\title{
Magnetic Resonance Imaging and machine learning make a valuable combined tool for the screening of preclinical AD
}

Paula Petrone $^{1}$, Verónica Vilaplana ${ }^{2}$, Adrià Casamitjana $^{2}$, Dalila Sanchez-Escobedo ${ }^{2}$, Alan Tucholka ${ }^{1,}$ Raffaele Cacciaglia ${ }^{1}$, Grégory Operto ${ }^{1}$, Stavros Skouras ${ }^{1}$, Carles Falcon ${ }^{1}$, José Luis Molinuevo ${ }^{1,3}$, Juan Domingo Gispert ${ }^{1}$

${ }^{1}$ Barcelonaßeta Brain Research Center, Pasqual Maragall Foundation, Barcelona, Spain.

${ }^{2}$ Department of Signal Theory and Communications, Technical University of Catalonia (UPC), 08034 Barcelona, Spain.

${ }^{3}$ Alzheimer's Disease and Other Cognitive Disorders Unit, Hospital Clínic, Institut d’Investigacions Biomediques August Pi i Sunyer (IDIBAPS), Barcelona, Spain

\section{Themes: Neuroimaging. Optimal neuroimaging measures for early detection}

Background: Worldwide efforts are turning to pharmacological prevention strategies to fight the dementia epidemic that confronts the next decades. However, prevention trials face huge ethical and logistic challenges. Efficient, cost-effective and noninvasive identification and follow-up assessment of subjects at the preclinical stages of Alzheimer's disease (AD) is currently an unmet medical need. We offer an efficient and minimally invasive pre-screening solution to identify subjects at risk of AD that would likely benefit from prevention strategies, based on advanced Magnetic Resonance Imaging and machine learning approaches.

Methods: Multimodal Magnetic Resonance Imaging (T13D, DWI and rs-fMRI) data was acquired from two independent cohorts with available CSF data that enabled biomarker based diagnostic classification: a local cohort from Hospital Clinic of Barcelona (HCB) with 87 subjects ( 40 controls, 12 preclinical, $21 \mathrm{MCI}$ due to AD, $14 \mathrm{AD}$ ) and a subset of the ADNI cohort comprising 96 subjects (28 controls, 16 preclinical, $23 \mathrm{MCI}$ due to $\mathrm{AD}, 29 \mathrm{AD}$ ) for which data is publicly available. We applied machine learning techniques to identify subjects at the preclinical stages of $\mathrm{AD}$, as well as other categories in the $\mathrm{AD}$ continuum. A receiving operator characteristic analysis was performed and we also estimated the economic impact of this pre-screening framework on recruitment in a typical prevention trial.

Results: Accuracy to detect the preclinical stage of AD achieved an AUC 0.85 and this performance was robust across both studied cohorts. Used as a pre-screening tool, this approach is estimated to save $50 \%$ of the expenses of clinical trial recruitment by sparing $80 \%$ invasive PET or CSF acquisitions from volunteers that are unlikely to be in the course of the disease. 
Conclusions: Our work establishes a proof of principle that pre-screening with MRI-based classifiers can significantly reduce clinical trial cost and lower the time and effort put by volunteers and caregivers. Our approach represents a valuable tool which shall foster public participation in prevention $\mathrm{AD}$ trials by minimizing invasive intervention.

\section{References:}

1. Morris, J.C. Early-stage and preclinical Alzheimer disease. Alzheimer Dis Assoc Disord. 2005; 19: 163-165. 2. Dubois, B., Feldman, H.H., Jacova, C., Hampel, H., Molinuevo, J.L., Blennow, K. et al. Advancing research diagnostic criteria for Alzheimer's disease: the IWG-2 criteria.Lancet Neurol. 2014; 13: 614-629.

3. Mueller SG, Weiner MW, Thal LJ, et al. Ways toward an early diagnosis in Alzheimer's disease: the Alzheimer's Disease Neuroimaging Initiative (ADNI) Alzheimers Dementia. 2005;1:55-66. 